\title{
Microscopy101
}

\section{Out of the Blue and into the Black: Preparation, Mounting, and Image Rendering of Complex, Chorate Dinoflagellate Cysts for Scanning Electron Microscopy}

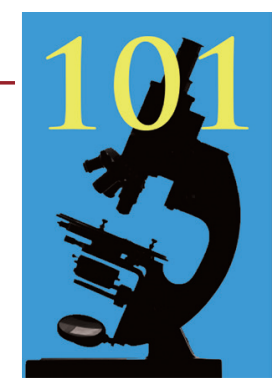

\author{
Sandy M.S. McLachlan ${ }^{1 \star}$ and Elaine C. Humphrey ${ }^{2}$ \\ ${ }^{1}$ School of Earth and Ocean Sciences, University of Victoria, PO Box 1700, STN CSC, V8W 2Y2, Victoria, BC, Canada \\ ${ }^{2}$ Advanced Microscopy Facility, Department of Mechanical Engineering, University of Victoria, PO Box 1700, STN CSC, \\ V8W 2Y2, Victoria, BC, Canada \\ *sandymcl@uvic.ca
}

\begin{abstract}
We describe an experimental approach for achieving an optimal black background for scanning electron photomicrographs of small samples with elaborate and intricate structures. Specimens of the highly ornate, 66-million-year-old chorate dinoflagellate cyst species Cannosphaeropsis franciscana were selected as the subject of this study. Photomicrographs collected following standard aluminum stub surface placement were compared to those taken of specimens mounted using a novel pin-and-pedestal method. This simplistic mounting technique minimizes the need for post-production image editing and extraneous background removal.
\end{abstract}

Keywords: dinoflagellate cysts, microfossils, SEM, specimen preparation, image post-production

\section{Introduction}

"And blood-black nothingness began to spin. A system of cells interlinked, within cells interlinked, within cells interlinked within one stem. And dreadfully distinct against the dark, a tall white fountain played." - Vladimir Nabokov, Pale Fire [1]

Clear presentation of data is the foundation of effective science communication. A classic image is that represented by the remarkable grayscale resolution of photomicrographs rendered through scanning electron microscopy (SEM). No greater is the stark definition revealed by this practice than that which can be perceived by the contrast between the lighter foreground features of the subject and a darker background. Scanning electron image rendering has come a long way toward achieving a dark background since the use of film [2]. However, attempts to engineer a sample holder suitable for the generation of a uniform black background with a suitable gray scale for foreground features have been limited [3], with emphasis in recent years having shifted to the now ubiquitous use of image editing software. A pin-and-pedestal method is proposed as a means for collecting an immaculate dark background to minimize the need for post-production image editing. Specimens of Cannosphaeropsis franciscana, a fossil species of organic-walled chorate dinoflagellate cyst of Late Cretaceousearly Paleocene age recovered from the sedimentary rocks of the Oyster Bay Formation on Vancouver Island, British Columbia, Canada [4], were selected as the subjects for this study. Their complex ornate structure, around which an undesirable bitmap background would otherwise pose a significant challenge for digital removal, makes them an ideal sample for this study.

\section{Materials and Methods}

Specimen recovery and preparation. The dinoflagellate cysts were prepared using a standardized extraction procedure [5]. Rock samples with a matrix of $\sim 2 \mathrm{~cm}^{3}$ were placed in $50 \mathrm{ml}$ polypropylene test tubes and treated repeatedly with $10 \%$ roomtemperature hydrochloric acid $(\mathrm{HCl})$ to dissolve any carbonates. Following $\mathrm{HCl}$ treatment, the samples were placed in $48 \%$ room-temperature hydrofluoric acid (HF) where they were left to sit with daily stirring for an average of two weeks (Figure 1). Each sample then underwent one round of $\mathrm{HCl}$ and two rinses of reverse osmosis water to remove any HF residue before final sieving through $120 \mu \mathrm{m}$ and $15 \mu \mathrm{m}$ Nitex nylon mesh to remove remaining particles. The samples were subjected to one minute of ultrasonic agitation to dislodge any minute sediment particles from the dinoflagellate cysts $[6,7]$ and centrifuged at $3600 \mathrm{rpm}$. Specimens prepared for SEM underwent up to an additional four minutes of ultrasonic agitation following sieving. A series of specimens were transferred to the surface of aluminum SEM stubs with a micropipette for standard presentation (Figure 2), while others were selected for mounting using the novel pinand-pedestal technique described herein.

The creation of a pin-and-pedestal is fairly straightforward and begins with the use of wire cutters to remove the $\sim 1 \mathrm{~mm}$ end of an entomological pin, which is optimal for its narrow, machined point. The pin tip is then gently touched to a carbon sticker to retain a minute bolus of sticker to hold the specimen in place. A small portion of carbon sticker is affixed to the stub surface into which the cut pin end is then embedded at a $90^{\circ}$ angle and reinforced with Paraloid B-72 adhesive as shown in Figure 3A. A dry dinoflagellate cyst specimen is then touched with a pin and transferred from a slide on the stage of a transmitted light microscope to the pin tip mounted vertically on the stub with the aid of a reflected light microscope. Once mounting was completed, the SEM stubs were coated with either gold or a gold/palladium mix using either an Edwards S150B or Anatech Hummer VI sputter coater for a thickness of $10-50 \mathrm{~nm}$. 


\section{TESCAN Dynamic micro-CT}

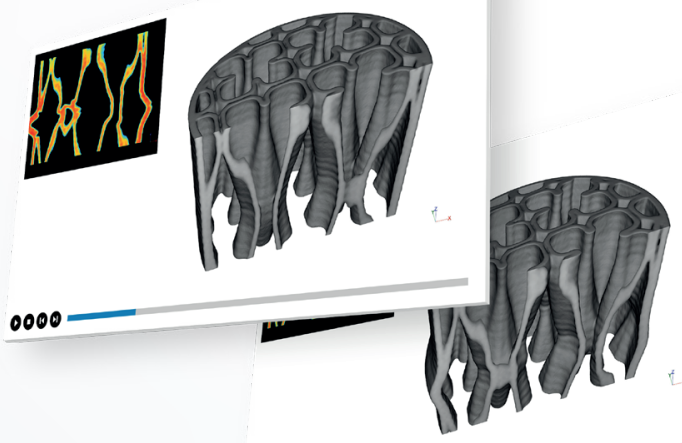

Leading the way in temporal resolution shifting your research from 3D to 4D

Dynamic Imaging of Aluminum Foam Compression - 20 sec/scan, 190 total scans
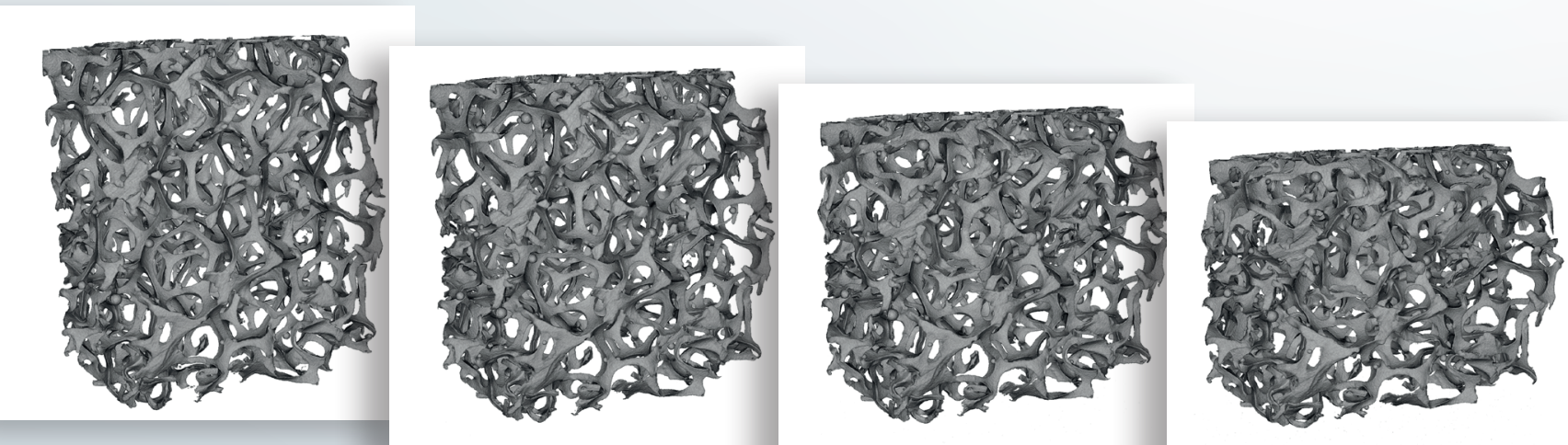

Dynamic Imaging of Dissolving Soap

Bubbles - $30 \mathrm{sec} / \mathrm{scan}, 150$ total scans

Analysis of Soap Bubble Coalescence
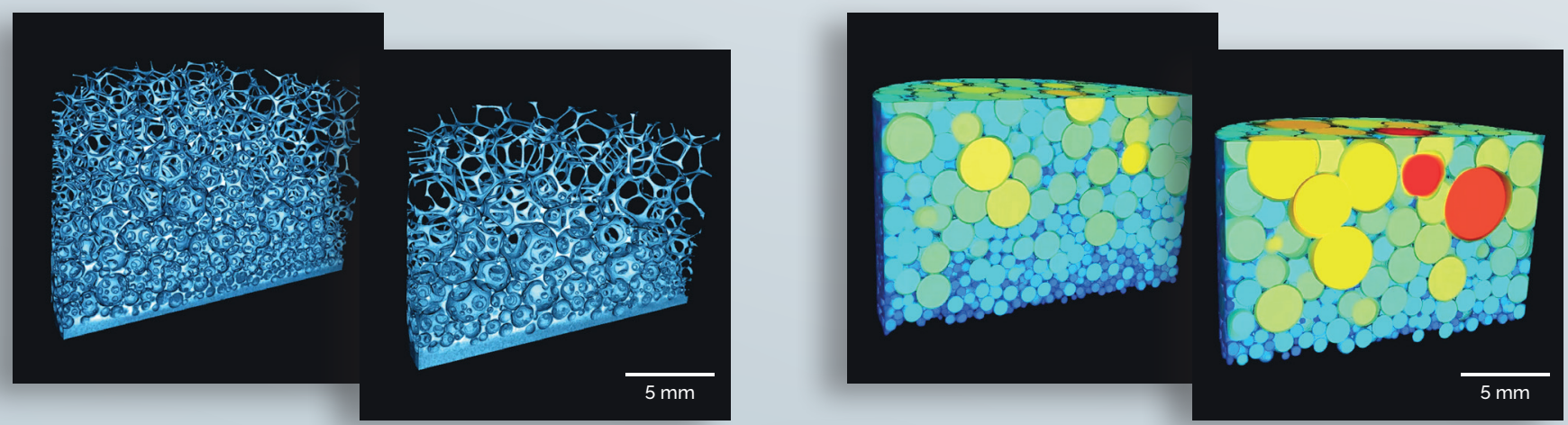

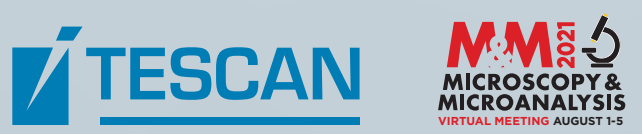

What can you do with Dynamic CT?

Contact us today to find out. WwW.tescan.com

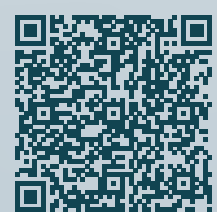




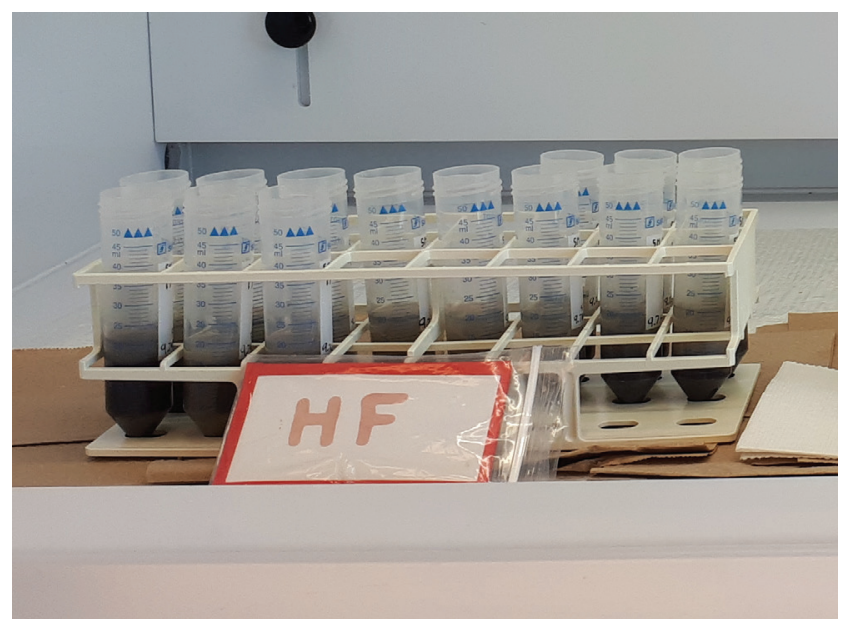

Figure 1: Sedimentary rock matrix undergoing hydrofluoric acid digestion to reveal organic microfossils at the Paleoenvironmental/Marine Palynology Laboratory, School of Earth and Ocean Sciences, University of Victoria.

Scanning electron image rendering. Micrographs of specimens were taken with a Hitachi field emission S-4800 SEM at the Advanced Microscopy Facility, University of Victoria (Victoria, British Columbia, Canada), using an accelerating voltage of $1 \mathrm{kV}$ and emission current of $10 \mu \mathrm{A}$. A series of images were taken at different focal points; as the focal point changed the magnification also changed. Once in focus, the magnification was adjusted so that the series of images were taken at the same magnification.

Image post-production. Initially, composite images were prepared for presentation using Helicon Focus, but the majority had to be manually combined using Adobe Photoshop CS6 or CC2019 software, putting the in-focus parts together in layers. Adjustments to noise using a Gaussian Blur and Unsharp Mask and Brightness/Contrast using Levels were occasionally necessary. When the pin-and-pedestal method was not employed and a black background was desired, the grayscale stub surface was typically changed through Adobe Photoshop using standard layer masks.

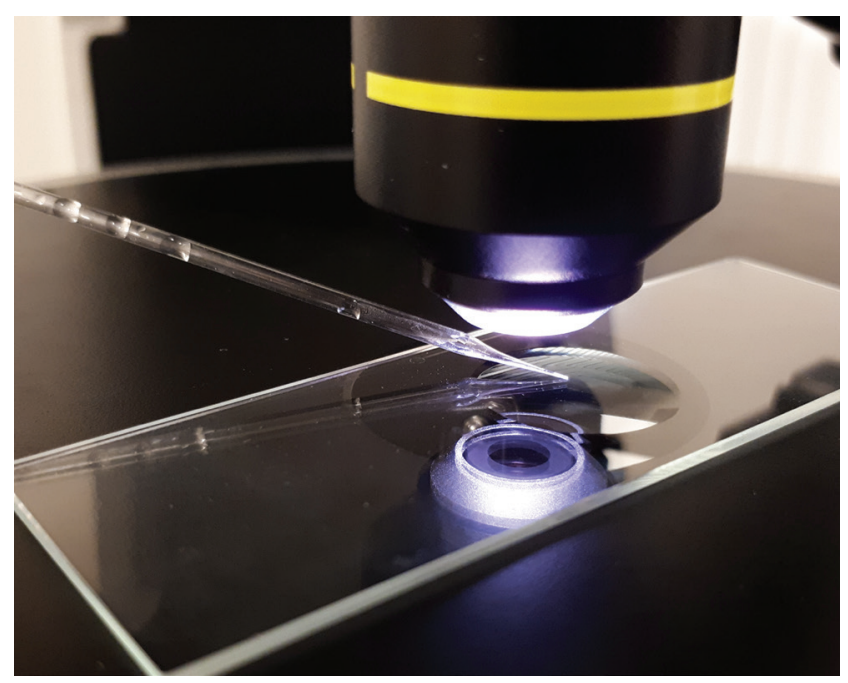

Figure 2: Use of a micropipette and transmitted light microscopy to transfer a Cannosphaeropsis franciscana dinoflagellate cyst specimen from a slide for placement on an SEM aluminum stub.
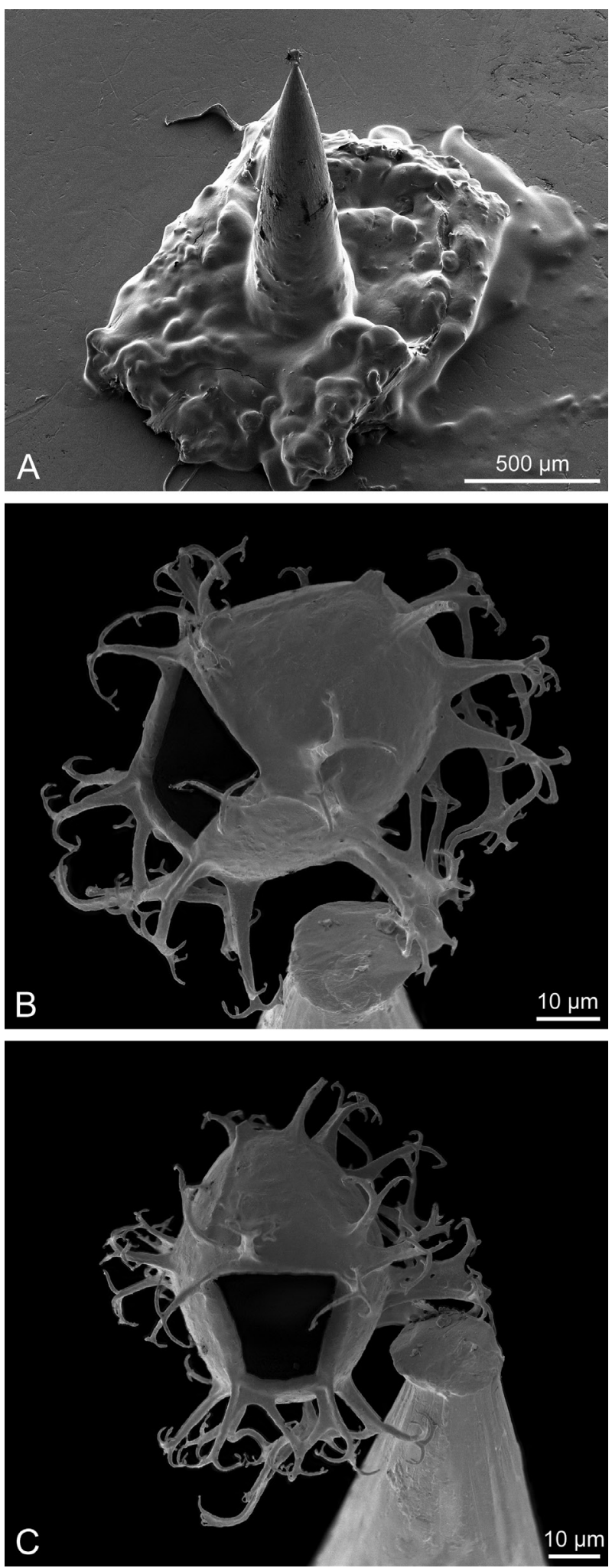

Figure 3: Pin-and-pedestal mount of a Cannosphaeropsis franciscana dinoflagellate cyst specimen. (A) Low-magnification view of a pin tip embedded in a carbon sticker on an aluminum stub surface. The pin is stabilized with Paraloid B-72 adhesive; (B, C) high-magnification views of a Cannosphaeropsis franciscana dinoflagellate cyst suspended on a pin tip. Note the minute bolus of carbon sticker on the pin tip to secure the specimen. The black background is shown as collected with the SEM and has not been altered by image processing. 


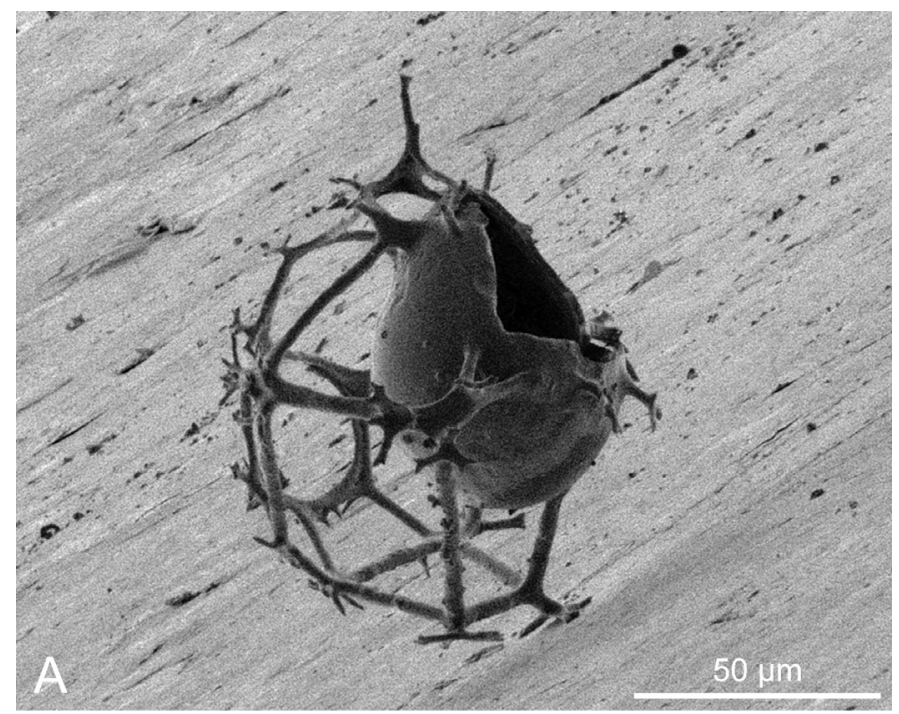

being mounted on a standard stub and is therefore capable of the full range of stage tilt and rotation.

When an ideal black background is required, the pin-andpedestal approach for preparation of small samples is a viable alternative to the Pohl specimen holder. While entirely feasible as demonstrated in this paper, mounting of microfossils may be daunting to execute and requires the utmost patience and fine motor function. In general use, the pin-and-pedestal technique may be most practical for the mounting of structures $>100 \mu \mathrm{m}$, provided enough carbon adhesive is applied to the pin tip. In the realm of micropaleontology, these include a wide range of microfossils of common interest such as conodont elements, foraminiferal tests, and radiolarian skeletons.

\section{References}

[1] VV Nabokov, Pale Fire: A Novel, G. P. Putnam's Sons, New York, 1962. ISBN 9780399121616.

[2] JE Whittaker and RL Hodgkinson, J Micropalaeontol 9 (1991) https://doi.org/10.1144/jm.9.2.219.

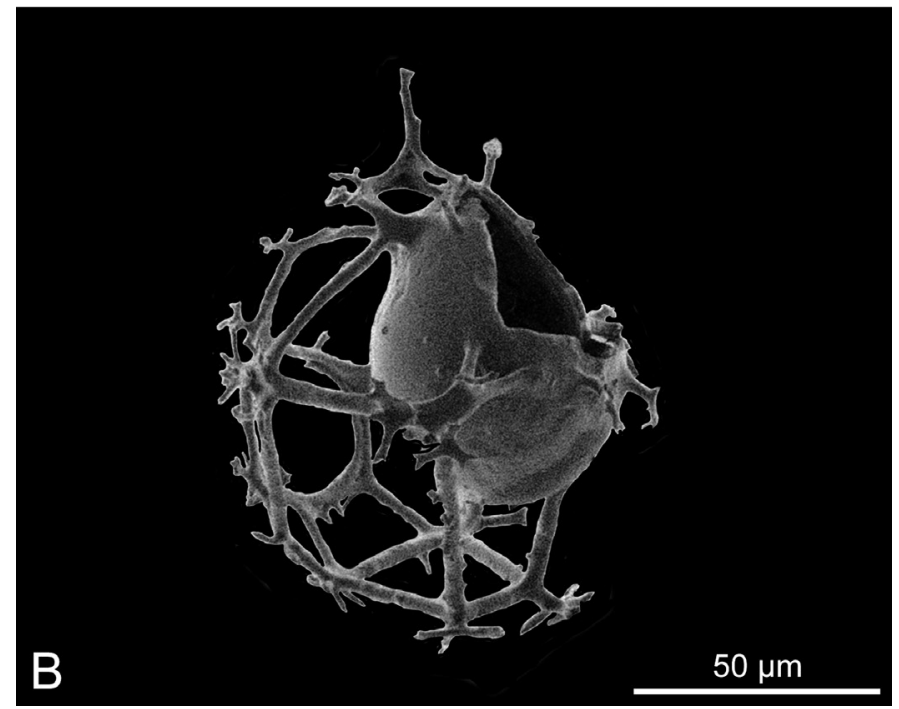

Figure 4: Scanning electron photomicrograph of a Cannosphaeropsis franciscana dinoflagellate cyst specimen with original and digitally replaced backgrounds. (A) Unaltered original photomicrograph of specimen placed on an aluminum stub surface; (B) same image with post-production Adobe Photoshop removal of the background replaced with black infill.

\section{Discussion}

Pohl's method, albeit more sophisticated in design than the pin-and-pedestal mount, requires a milled pot cavity backdrop, platform, and screw pin mount. Equivalent image quality is achieved with Pohl's apparatus, but it is more cumbersome in its application and requires the manufacture and assembly of multiple parts (see Figure 1 in [3]). In addition, Pohl's specimen holder was designed for the purpose of imaging arthropod specimens (see Figure 2 in [3]) that are orders of magnitude larger than the dinoflagellate cysts examined in this study. Pohl's holder also requires that samples be adhered to a pin with nail polish that would result in smaller structures, such as most microfossils, being engulfed by the medium. Additionally, Pohl's pin-and-pot configuration requires the specimen to be fixed at one viewing angle at a time. This requires that the sample be removed from the SEM when any stage adjustment is required, whereas the pin-and-pedestal mount benefits from

[3] H Pohl, Microsc Res Tech 73 (2010) https://doi.org/10.1002/ jemt.20835.

[4] SMS McLachlan et al., Rev Palaeobot Palynol 292 (2021) https://doi.org/10.1016/j.revpalbo.2021.104452.

[5] V Pospelova et al., Marine Micropaleontol 75 (2010) https://doi.org/10.1016/j.marmicro.2010.02.003.

[6] KN Mertens et al., Rev Palaeobot Palynol 184 (2012) https://doi.org/10.1016/j.revpalbo.2012.06.012.

[7] AM Price et al., Rev Palaeobot Palynol 226 (2016) https:// doi.org/10.1016/j.revpalbo.2015.12.009.

\section{TGS Technologies, LLC TEM Sample Holder Service and Modification Specialist}

With over 40 years of experience repairing TEM Sample Holders, we provide you with high-quality personalized service. We specialize in repair, modification, and refurbishment of your sample holder as well as custom design if you are seeking specific innovative needs from your equipment.

TGS Technologies, LLC

Ph: 724-453-3865

Fx: 724-453-2968

Email: tom@tgstechnologies.net http://www.tgstechnologies.net 